
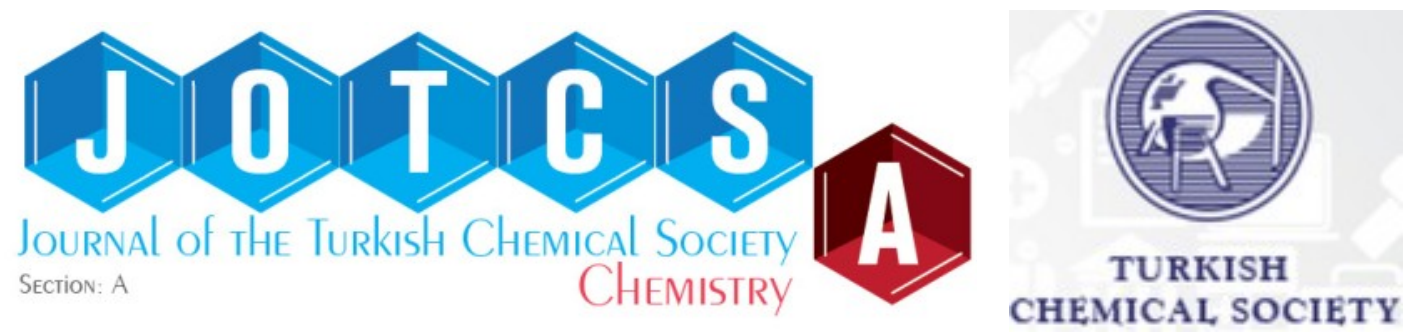

\title{
Fabrication of Superhydrophobic Coatings on Glass based on Poly(dimethylsiloxane) and Fumed Silica
}

\author{
Esra KASAPGİL $\triangle D$, Alper NERAT $\square$, Buse M. POYRAz $\square$ iD, ílke ANAÇ* \\ Department of Materials Science and Engineering, Gebze Technical University, Gebze, Kocaeli, Turkey.
}

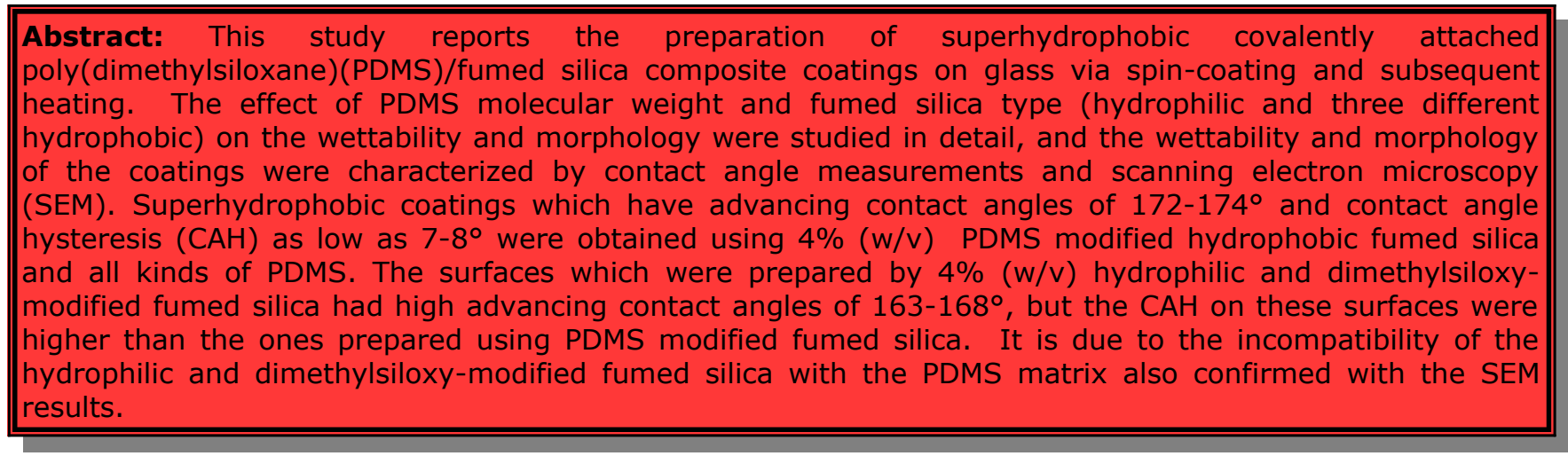

Keywords: poly(dimethylsiloxane), fumed silica, wetting, superhydrophobic coatings, contact angle, spincoating

Submitted: February 21, 2020. Accepted: June 07, 2020.

Cite this: KASAPGİL E, NERAT A, POYRAZ BM, ANAÇ İ. Fabrication of Superhydrophobic Coatings on Glass based on Poly(dimethylsiloxane) and Fumed Silica. JOTCSA. 2020;7(2):589-96.

DOI: https://doi.org/10.18596/jotcsa.692565.

*Corresponding author. E-mail: ilkeanac@gtu.edu.tr

\section{INTRODUCTION}

There is a great interest in the fabrication of artificial superhydrophobic surfaces over the last 20 years after Barthlott et al. reported the self-cleaning property of a lotus leaf in 1997, which is known as a "lotus effect" (1-5). Superhydrophobic surfaces, which have water contact angles (CA) larger than $150^{\circ}$, sliding angles lower than $5^{\circ}$ or contact angle hysteresis (CAH) lower than $10^{\circ}$, can be used in various applications such as oil-water separation, self-cleaning, anti-icing, anti-fouling, and drop transport (5-7). There are two crucial factors when designing hydrophobic surfaces; (1) using a low energy material as a coating and (2) creating a rough surface, which will increase the hydrophobic surface character according to the Cassie Baxter wetting situation (8). Therefore, superhydrophobic surfaces can be prepared either by modifying rough surfaces using low energy materials or by making a roughness using low energy materials $(2-7,9)$.

To date, researchers used different techniques to create various superhydrophobic surfaces such as sol-gel, lithography, templating method, colloidal assembling and aggregation, layer-by-layer assembly, plasma method, chemical vapor deposition, phase separation, electrospinning and electrospraying, dip-coating and so on $(4,10-11)$. These techniques are quite complicated and expensive due to multiple steps, specific equipment usage, and costly reagents. Besides, they can be applied to specific substrates. Overcoming such difficulties, different kinds of polymers, together with various nanoparticles, were used to fabricate superhydrophobic surfaces on different substrates where the nanoparticles were used to create surface texturing on the surfaces (12-18). Silica-based 
nanofillers are the most used ones in the literature to create superhydrophobic and self-cleaning surfaces (14, 19-26). Manoudis et al. prepared superhydrophobic composite films containing hydrophilic silica nanoparticles and poly(methyl methacrylate) (PMMA) and commercial poly(alkyl siloxane) (Rhodorsil 224) on various substrates (glass, silicon, wood, concrete, aluminum, silk, marble) via spray coating (14). Superhydrophobic films were fabricated on glass via a drop-coating method using Sylgard 184 silicone (containing curing agent and poly(dimethylsiloxane) (PDMS) prepolymer) and silica particles (19). Chang et al. successfully prepared the superhydrophobic wood surfaces via a dip-coating method using PDMS Syglard 184, curing agent, and hydrophobic silica particles (20). They also investigated the morphology, wetting behavior, and roughness of the coatings as a function of silica concentration (20). Superhydrophobic composite films containing hydrophobic fumed silica particles and different polymers (thermoplastic resin, PS, cross-linked epoxy resin, PDMS-urea copolymer, polyether-based polyurethane urea, PMMA, polycarbonate) were prepared on glass via spin-coating process or doctor blade coating method by Yilgor and coworkers (2123). Cai et al. fabricated superhydrophobic fabrics via successive dip-coating of PDMS and silica suspensions and successive annealing steps (24). Also, bioinspired superhydrophobic cross-linked polyurethane-acrylate coatings containing hydrophobic fumed silica on glass were prepared via bar coating following UV curing by Fourmentin and coworkers (25). The superhydrophobic coating on cellulosic filter paper for oil-water separation was achieved via solution casting technique using PMMA copolymer and silica nanoparticles by Siriam et al. (26).

As noted above, several papers in the literature report the preparation of superhydrophobic coatings on various substrates such as glass, wood, aluminum, silicon wafer using PDMS, low surface energy material, and nanoparticles $(14,19,20,24,27)$. Either these coatings are not covalently attached to the substrates (14), or they are prepared using curing agents $(19,20,24,27)$. In 2011, McCarthy and coworkers showed, for the first time, that linear polysiloxanes containing only Si-O$\mathrm{Si}$ in their main chain react with inorganic surfaces containing hydroxyl groups upon heating to desired temperature (especially $100{ }^{\circ} \mathrm{C}$ for $24 \mathrm{~h}$ ) (28). Furthermore, they reported that they obtained low hysteresis surfaces and concluded that they could control the thickness of the PDMS layers on silica by changing the molecular weight of the PDMS.

In the present study, we have prepared superhydrophobic PDMS/silica coatings which are covalently attached to glass via a simple spincoating process and subsequent heating. The wettability and surface morphology of PDMS/silica composites on glass were characterized by contact angle measurements (equilibrium, advancing and receding contact angle) and scanning electron microscopy (SEM), respectively. The effect of PDMS molecular weight, silica concentration, and silica type on coatings' morphology and contact angles were also systematically studied, and the results were compared with the findings reported in the literature.

\section{EXPERIMENTAL DETAILS}

\section{Materials}

Polydimethylsiloxane (PDMS) with three different weight average molecular weight $\left(M_{w}\right)$ of $2000(2 k)$, $63000(63 \mathrm{k})$ and $117000(117 \mathrm{k})$ were obtained from Alfa Aesar (Massachusetts, USA). Amorphous hydrophobic fumed silicon (IV) oxide ( $\sim 50 \mathrm{~nm}$; PDMS modified, referred to as FS) was obtained from Alfa Aesar. Amorphous hydrophobic pyrogenic silica with different particle sizes and different surface modification ( $40 \mathrm{~nm}$; dimethylsiloxy modified; referred as $\mathrm{H} 15$ and $\sim 30 \mathrm{~nm}$; PDMS modified; referred as $\mathrm{H} 18$ ) and amorphous hydrophilic pyrogenic silica ( $\sim 25 \mathrm{~nm}$; referred as N20) were obtained from Wacker (Burghausen, Germany). Toluene was obtained from SigmaAldrich. Ethanol, sulfuric acid $\left(\mathrm{H}_{2} \mathrm{SO}_{4}, 95-97 \%\right)$, hydrogen peroxide $\left(\mathrm{H}_{2} \mathrm{O}_{2}, 30 \%\right)$, and ultra-pure water were obtained from Merck (Darmstadt, Germany). All reagents were used as received without further purification. Glass microscope slides were obtained from ISOLAB GmbH (Wertheim, Germany) and cut into $25 \mathrm{~mm} \times 35 \mathrm{~mm} \times 1 \mathrm{~mm}$ pieces. Deionized water (18.2 $\left.\mathrm{M} \Omega \mathrm{cm}^{-1}\right)$ was obtained through a system including pretreatment, reverse osmosis, and ion exchange steps (Elga Option Q15).

\section{Preparation of PDMS-Silica Coatings}

Glass microscope slides were cleaned by ethanol and then activated by piranha solution before the coating procedure. Glass slides were immersed into freshly prepared piranha solution, which is a 7:3 (v:v) mixture of concentrated $\mathrm{H}_{2} \mathrm{SO}_{4}$ and $30 \% \mathrm{H}_{2} \mathrm{O}_{2}$. They were kept at $80{ }^{\circ} \mathrm{C}$ for $2 \mathrm{~h}$ and then left at room temperature overnight. Then the glass slides were washed with deionized water and dried at 125 ${ }^{\circ} \mathrm{C}$ for $30 \mathrm{~min}$ in an oven. Activated glass slides were used immediately for PDMS-silica composite film preparation.

The coating solution was prepared as follows; the desired amount of silica particles (to obtain $\mathrm{x} \% \mathrm{w} / \mathrm{v}$ for the toluene volume, where $\mathrm{x}$ is $0,0.4,1.2,2,3$, and 4 ) were added to $1 \% \mathrm{w} / \mathrm{v}$ PDMS toluene solution. The polymer solution was mixed with a magnetic stirrer for $1 \mathrm{~h}$ and then sonicated for 30 min at room temperature. $10 \mu \mathrm{L}$ of the homogeneous polymer solution was dropped onto the freshly activated glass slide and spin-coated at 
$2000 \mathrm{rpm}$ for $60 \mathrm{~s}$. Then the coatings were kept at $100{ }^{\circ} \mathrm{C}$ for $24 \mathrm{~h}$ in an oven (Figure 1).

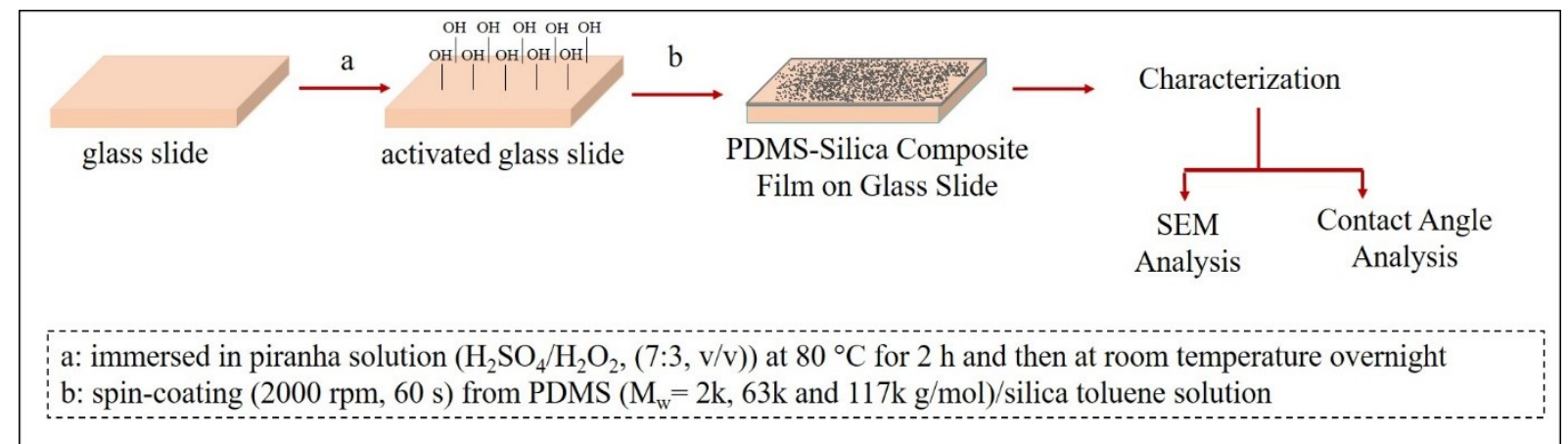

Figure 1: Schematic illustration of the PDMS-silica composite film preparation and characterization procedure.

\section{Characterization of The Coatings}

The surface morphology of the coatings was characterized by scanning electron microscopy (SEM, Philips XL 30 SFEG). PDMS-silica composite films were coated with a gold layer before SEM analysis to prevent charging effects. Contact angles were measured by a contact angle meter (KSV-CAM 200) with a PC controlled motorized syringe using ultra-pure water under air at room temperature. Static contact angles $\left(\theta_{\mathrm{e}}\right)$ were measured by forming $5 \mu \mathrm{L}$ drops of water on samples, and the values recorded $2 \mathrm{~s}$ after the release of the needle. Advancing contact angles $\left(\theta_{a}\right)$ were measured by increasing the volume of the water droplet on the surface from 3 to $8 \mu \mathrm{L}$, and receding contact angles $\left(\Theta_{r}\right)$ were measured by decreasing the volume of a water droplet from 10 to $3 \mu \mathrm{L}$ by using the automatic dispenser through the needle. The needle was kept within the water droplet during the $\Theta_{a}$ and $\Theta_{r}$ measurements. The reported values were calculated by taking the averages of 5 different measurements made on the same sample.

Chemical stabilities of PDMS/silica composite coatings were tested by washing the samples with toluene for 5 minutes and dried at $100{ }^{\circ} \mathrm{C}$ for $24 \mathrm{~h}$ in the oven. SEM images were again taken, and contact angle $\left(\Theta_{e}, \theta_{a}\right.$, and $\left.\theta_{r}\right)$ values were again measured after washing with toluene, and the results were compared with the unwashed samples' results.

\section{RESULTS AND DISCUSSION}

In this study, covalently attached superhydrophobic PDMS/silica coatings were prepared on piranhacleaned glass slides. The superhydrophobic coatings were prepared via a spin-coating of silica/PDMS dispersions and subsequent heating at $100{ }^{\circ} \mathrm{C}$ for 24 h. The covalent attachment was achieved via heating, as reported in the literature by McCarthy et al. (28). PDMS with three different molecular weights, hydrophilic, and three different hydrophobic fumed silica were used to prepare the coatings. The effect of hydrophobic fumed silica concentration on surface morphology and wettability were also studied.

Table 1 shows the effect of fumed silica concentration on the equilibrium $\left(\Theta_{\mathrm{e}}\right)$, advancing $\left(\Theta_{a}\right)$ and receding $\left(\Theta_{\mathrm{r}}\right)$ contact angle and contact angle hysteresis (CAH) values of composite films. The composites films were prepared by two different molecular weights $(63 \mathrm{k}$ and $117 \mathrm{k} \mathrm{g} / \mathrm{mol}$ ) of PDMS and five different concentration of hydrophobic fumed silica $(0.4,1.2,2,3$ and $4 \% \mathrm{w} / \mathrm{v})$ where hydrophobicity is achieved by modification with PDMS ( $\sim 50 \mathrm{~nm}$; referred as, FS). As seen in Table 1 , the $\theta_{e}, \theta_{a}$ and $\theta_{r}$ values of bare PDMS coating increase from $108 \pm 1^{\circ}, 111 \pm 1^{\circ}$ and $96 \pm 2^{\circ}$ to $119 \pm 1^{\circ}, 120 \pm 1^{\circ}$ and $103 \pm 2^{\circ}$ respectively as the molecular weight of PDMS increases from $63 \mathrm{k}$ to $117 \mathrm{k}$. This trend is similar to the results reported in the literature [28]. The $\theta_{e}, \theta_{a}$, and $\theta_{r}$ values of the coatings increased with an introduction of hydrophobic fumed silica (FS) as depicted in Table 1 regardless of PDMS molecular weight. As the concentration of hydrophobic fumed silica (FS) increased from 0.4 to $4 \% \mathrm{w} / \mathrm{v}$, and increase in $\Theta_{\mathrm{e}}$, $\Theta_{a}$ and $\theta_{r}$ values were seen up to $169 \pm 2^{\circ}, 173 \pm 1^{\circ}$, $166 \pm 1^{\circ}$, and $170 \pm 2^{\circ}, 172 \pm 2^{\circ}$ and $164 \pm 1^{\circ}$ for the coatings prepared by $63 \mathrm{k}$ and $117 \mathrm{k}$ PDMS respectively (Table 1 ). The highest contact angle values and lowest $\mathrm{CAH}$ ( $7^{\circ}$ for 63k PDMS and $8^{\circ}$ for $117 \mathrm{k}$ PDMS) were obtained at $4 \% \mathrm{w} / \mathrm{v}$ silica concentration for PDMS coatings prepared by different molecular weights. The highest $\theta_{e}$ value $\left(164^{\circ}\right)$ of cross-linked silicone-based coatings prepared by spray coating using $\mathrm{CaCO}_{3} / \mathrm{SiO}_{2}$ particles on glass by Yang et al. (27) was lower than our results. Manoudis et al. reported that they obtained siloxane-based coatings via spraying on glass using $2 \% \mathrm{w} / \mathrm{v}$ hydrophilic silica particles with a $\Theta_{a}$ of $164^{\circ}$ and $\mathrm{CAH}$ of $3^{\circ}$. The CAH hysteresis value $\left(3^{\circ}\right)$ is better than our values ( $7^{\circ}$ for 63k PDMS and $8^{\circ}$ for $117 k$ PDMS), but the information on the chemical composition and the molecular weight of the poly(alkyl siloxane) which was used to prepare the coating was not given in the paper (14). 
Table 1. Equilibrium $\left(\Theta_{\mathrm{e}}\right)$, advancing $\left(\Theta_{\mathrm{a}}\right)$ and receding $\left(\Theta_{\mathrm{r}}\right)$ contact angle and contact angle hysteresis $(\mathrm{CAH})$ values of $\mathrm{PDMS} / \mathrm{SiO}_{2}$ composite films prepared by hydrophobic fumed silica (FS) and PDMS with different molecular weights. The PDMS (1\% w/v) was prepared by dissolving PDMS (63k or $117 \mathrm{k})$ in toluene.

\begin{tabular}{|c|c|c|c|c|c|}
\hline $\begin{array}{c}\text { PDMS } \\
\left(M_{w}(g / m o l)\right)\end{array}$ & $\begin{array}{c}\text { Silica/toluene } \\
\text { ( } \% \mathrm{w} / \mathrm{v})\end{array}$ & $\theta_{e}\left(^{0}\right)$ & $\theta_{\mathbf{a}}\left(^{\circ}\right)$ & $\theta_{r}\left(^{0}\right)$ & CAH $\left(^{\circ}\right)$ \\
\hline $63 k$ & 0 & $108 \pm 1$ & $111 \pm 1$ & $96 \pm 2$ & 15 \\
\hline $63 k$ & 0.4 & $119 \pm 2$ & $124 \pm 1$ & $108 \pm 2$ & 16 \\
\hline $63 k$ & 1.2 & $161 \pm 1$ & $168 \pm 2$ & $150 \pm 1$ & 18 \\
\hline $63 k$ & 2 & $167 \pm 1$ & $171 \pm 1$ & $155 \pm 2$ & 16 \\
\hline $63 k$ & 3 & $169 \pm 1$ & $172 \pm 2$ & $164 \pm 1$ & 8 \\
\hline $63 \mathrm{k}$ & 4 & $169 \pm 2$ & $173 \pm 1$ & $166 \pm 1$ & 7 \\
\hline $117 \mathrm{k}$ & 0 & $119 \pm 1$ & $120 \pm 1$ & $103 \pm 1$ & 17 \\
\hline $117 \mathrm{k}$ & 0.4 & $129 \pm 2$ & $146 \pm 1$ & $107 \pm 1$ & 39 \\
\hline $117 k$ & 1.2 & $166 \pm 2$ & $167 \pm 1$ & $142 \pm 1$ & 25 \\
\hline $117 \mathrm{k}$ & 2 & $167 \pm 1$ & $168 \pm 1$ & $134 \pm 1$ & 34 \\
\hline $117 \mathrm{k}$ & 3 & $169 \pm 2$ & $172 \pm 1$ & $159 \pm 2$ & 13 \\
\hline $117 k$ & 4 & $170 \pm 2$ & $172 \pm 2$ & $164 \pm 1$ & 8 \\
\hline
\end{tabular}

The $\theta_{e}, \theta_{a}, \theta_{r}$, and CAH values of bare PDMS and composite coatings before and after washing with toluene were shown in Table 2. The coatings were prepared using PDMS with different molecular weights $(2 \mathrm{k}, 63 \mathrm{k}$, and $117 \mathrm{k})$ and $4 \% \mathrm{w} / \mathrm{v}$ hydrophobic fumed silica (FS). As seen in Table 2, the $\theta_{\mathrm{e}}, \theta_{\mathrm{a}}, \theta_{\mathrm{r}}$ values of the bare $2 \mathrm{k}$ PDMS and $63 \mathrm{k}$ PDMS coatings were slightly decreased $\left(1-2^{\circ}\right)$ or increased $\left(1-2^{\circ}\right)$ after washing with toluene, but the decrease in $\Theta_{e}, \Theta_{a}, \Theta_{r}$ values of the coating prepared using $117 \mathrm{k}$ PDMS were higher compared to the other coatings. The increase in all the contact angle values was seen as the molecular weight of the PDMS rises, as shown in Table 2, and the trend did not change after washing with toluene. Similar to the bare PDMS coatings, there was a slight change $\left(1-2^{\circ}\right)$ in the $\theta_{e}, \theta_{a}, \theta_{r}$ values of the composite coatings prepared using $4 \% \mathrm{w} / \mathrm{v}$ silica (FS) after washing with toluene. The results in Table 2 clearly show that bare PDMS and composite coatings were covalently attached to the piranha cleaned glass slides, and the superhydrophobic character of the composite coatings did not alter after washing with toluene.

Figure 2 shows the SEM images of the coatings prepared by PDMS with different molecular weights PDMS/silica (FS) composite before and after washing with toluene. All the SEM pictures in Figure 2 a-c clearly show that the homogenous PDMS/silica (FS) composite coatings were obtained with a similar morphology regardless of the PDMS molecular weight. No change in the homogeneity of the composite coatings was observed after washing with toluene. (Figure $2 \mathrm{~d}-\mathrm{f}$ ).

After investigating the effect of silica concentration on coatings' wettability, $4 \%$ w/v silica concentration and 63k PDMS were chosen to study the effect of silica size and type on coating morphology and wettability (Table 3 and Figure 3 ). The $\theta_{e}, \Theta_{a}, \Theta_{r}$, and CAH values of PDMS/silica composite films prepared using different types and sizes of fumed silica were shown in Table 3 . The $\theta_{e}$ contact angles of the PDMS coating increased up to $159-172^{\circ}$ with an addition of different types and sizes of fumed silica (Table 3). Although high $\Theta_{a}$ values were obtained for the coatings prepared by hydrophilic fumed silica (N2O) $\left(168 \pm 2^{\circ}\right)$ and dimethylsiloxy modified hydrophobic fumed silica (H15) (N2O) $\left(163 \pm 2^{\circ}\right)$, the surfaces were not superhydrophobic due to the high $\mathrm{CAH}$ hysteresis ( $24^{\circ}$ and $16^{\circ}$ respectively). Superhydrophobic composite coatings were obtained when two different sizes of PDMS modified fumed silica particles (FS; 50 nm and $\mathrm{H18} ; \sim 30 \mathrm{~nm}$ ) were used to prepare the coatings. The $\Theta_{a}, \Theta_{r}$, and $\mathrm{CAH}$ values of the coatings prepared using $\mathrm{FS}$ and $\mathrm{N} 18$ were measured as $173 \pm 1^{\circ}, 166 \pm 1^{\circ}, 7^{\circ}$, and $174 \pm 1^{\circ}$, $166 \pm 1^{\circ}, 8^{\circ}$ respectively. These differences can be due to the incompatibility of the hydrophilic (N20) and dimethylsiloxy modified hydrophobic (H15) 
Table 2. Equilibrium $\left(\Theta_{\mathrm{e}}\right)$, advancing $\left(\Theta_{\mathrm{a}}\right)$ and receding $\left(\Theta_{\mathrm{r}}\right)$ contact angle and contact angle hysteresis $(\mathrm{CAH})$ values of $\mathrm{PDMS} / \mathrm{SiO}_{2}$ composite before and after washing with toluene. The composite films composed of hydrophobic fumed silica (FS) and PDMS with different molecular weights. The PDMS (1\% w/v) was prepared by dissolving PDMS $(2 \mathrm{k}, 63 \mathrm{k}$, or $117 \mathrm{k})$ in toluene.

\begin{tabular}{|c|c|c|c|c|c|c|}
\hline $\begin{array}{c}\text { PDMS } \\
\left(\mathrm{M}_{\mathrm{w}}(\mathrm{g} / \mathrm{mol})\right)\end{array}$ & $\begin{array}{c}\text { Silica/toluene } \\
(\% \mathrm{w} / \mathrm{v})\end{array}$ & Washing & $\theta_{\mathrm{e}}\left({ }^{\circ}\right)$ & $\theta_{a}\left({ }^{\circ}\right)$ & $\theta_{r}\left({ }^{\circ}\right)$ & CAH $\left({ }^{\circ}\right)$ \\
\hline $2 \mathrm{k}$ & - & No & $94 \pm 1$ & $95 \pm 2$ & $81 \pm 1$ & 14 \\
\hline $2 k$ & - & Toluene & $92 \pm 2$ & $99 \pm 2$ & $82 \pm 1$ & 17 \\
\hline $2 k$ & 4 & No & $170 \pm 1$ & $173 \pm 2$ & $166 \pm 1$ & 7 \\
\hline $2 k$ & 4 & Toluene & $168 \pm 1$ & $172 \pm 1$ & $162 \pm 1$ & 10 \\
\hline $63 \mathrm{k}$ & - & No & $108 \pm 1$ & $111 \pm 1$ & $96 \pm 2$ & 15 \\
\hline $63 k$ & - & Toluene & $109 \pm 1$ & $110 \pm 1$ & $96 \pm 1$ & 14 \\
\hline $63 k$ & 4 & No & $169 \pm 2$ & $173 \pm 1$ & $166 \pm 1$ & 7 \\
\hline $63 \mathrm{k}$ & 4 & Toluene & $168 \pm 1$ & $172 \pm 1$ & $165 \pm 2$ & 7 \\
\hline $117 k$ & - & No & $119 \pm 1$ & $120 \pm 1$ & $103 \pm 1$ & 17 \\
\hline $117 \mathrm{k}$ & - & Toluene & $110 \pm 2$ & $117 \pm 1$ & $98 \pm 1$ & 19 \\
\hline $117 \mathrm{k}$ & 4 & No & $170 \pm 2$ & $172 \pm 2$ & $164 \pm 1$ & 8 \\
\hline $117 \mathrm{k}$ & 4 & Toluene & $171 \pm 2$ & $173 \pm 2$ & $165 \pm 1$ & 8 \\
\hline
\end{tabular}



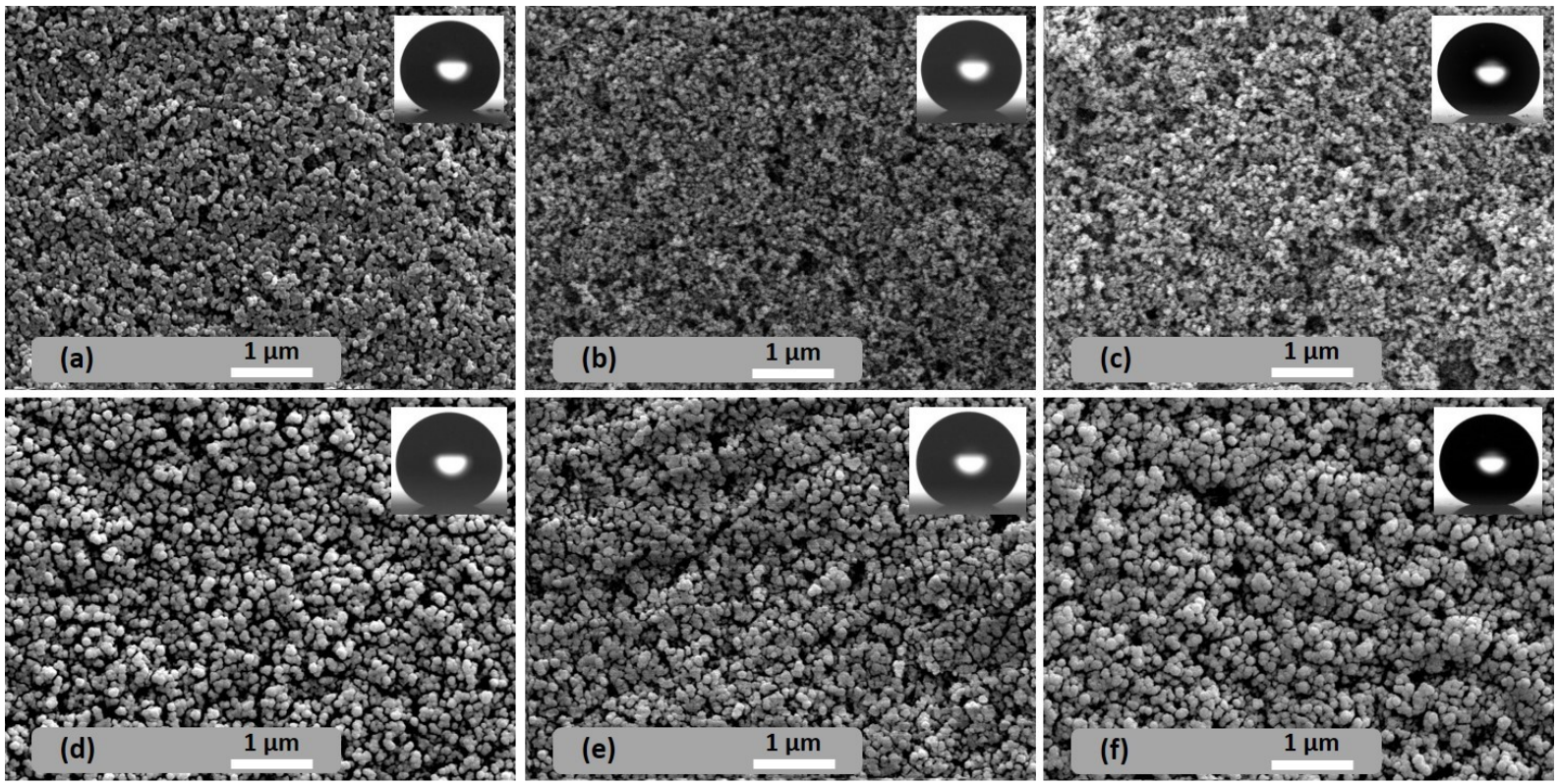

Figure 2. SEM pictures and water droplets of PDMS-silica composite films prepared with fumed silica (FS) and PDMS with different molecular weights : (a) 2k PDMS; (b) 63k PDMS; (c) 117k PDMS; (d) 2k PDMS, washed with toluene; (e) 63k PDMS, washed with toluene; and (f) 117k PDMS, washed with toluene (Silica concentration is $4 \% \mathrm{w} / \mathrm{v}$ ).

Table 3. Equilibrium $\left(\Theta_{\mathrm{e}}\right)$, advancing $\left(\Theta_{\mathrm{a}}\right)$ and receding $\left(\Theta_{\mathrm{r}}\right)$ contact angle and contact angle hysteresis $(\mathrm{CAH})$ values of $\mathrm{PDMS} / \mathrm{SiO}_{2}$ composite films with hydrophilic and three different hydrophobic fumed silica and PDMS (63k). The PDMS (1\% w/v) was prepared by dissolving PDMS (63k) in toluene.

\begin{tabular}{cccccc}
\hline Silica type & $\begin{array}{c}\text { Silica/toluene } \\
(\% \mathbf{\%} / \mathbf{v})\end{array}$ & $\boldsymbol{\theta}_{\mathbf{e}}\left(^{\circ}\right)$ & $\boldsymbol{\theta}_{\mathbf{a}}\left(^{\circ}\right)$ & $\boldsymbol{\theta}_{\mathbf{r}}\left(^{\circ}\right)$ & $\mathbf{C A H}^{\circ}\left(^{\circ}\right)$ \\
\hline- & - & $108 \pm 1$ & $111 \pm 1$ & $96 \pm 2$ & 15 \\
$\mathrm{FS}$ & 4 & $169 \pm 2$ & $173 \pm 1$ & $166 \pm 1$ & 7 \\
$\mathrm{~N} 20$ & 4 & $163 \pm 2$ & $168 \pm 2$ & $144 \pm 2$ & 24 \\
$\mathrm{H} 15$ & 4 & $159 \pm 1$ & $163 \pm 1$ & $147 \pm 1$ & 16 \\
$\mathrm{H} 18$ & 4 & $172 \pm 2$ & $174 \pm 1$ & $166 \pm 1$ & 8 \\
\hline
\end{tabular}

SEM pictures of the PDMS/fumed silica coatings which were prepared using $4 \% \mathrm{w} / \mathrm{v}$ PDMS modified hydrophobic fumed silica (FS and $\mathrm{H} 18$ ) and dimethylsiloxy modified fumed silica ( $\mathrm{H} 15)$ and hydrophilic fumed silica (N2O) are depicted in Figure $3 a, b, c$, and d respectively. Figure 3 a and b shows that the coatings prepared using FS and N10 are homogenous and very small aggregates of the fumed silica were seen on the surfaces confirming the compatibility of the PDMS modified fumed silica with the PDMS matrix. Distinct big aggregates of fumed silica, which is embedded in the PDMS matrix and non-homogeneous coating formation were observed as seen in Figure $2 \mathrm{c}$ and $\mathrm{d}$ when dimethylsiloxy modified fumed silica (H15) and hydrophilic fumed silica (N2O) were used conforming the non-compatibility of the particles with the PDMS matrix. 
I.
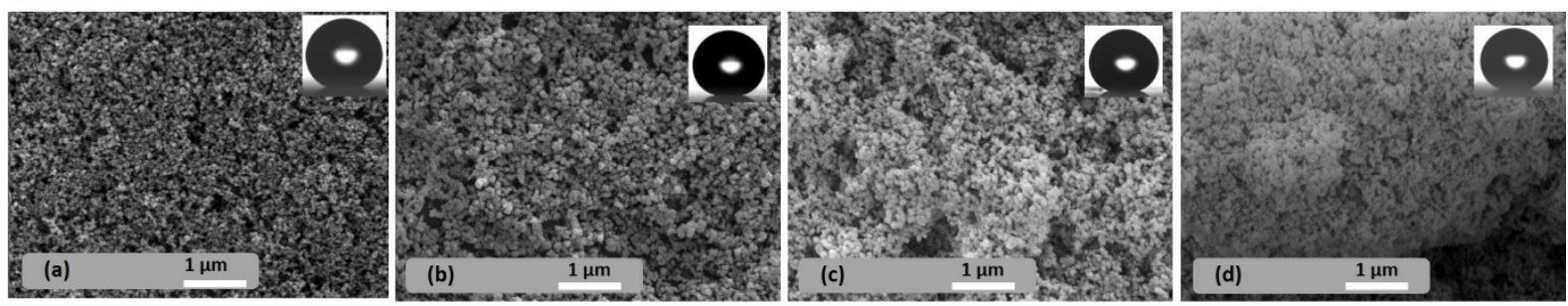

II.

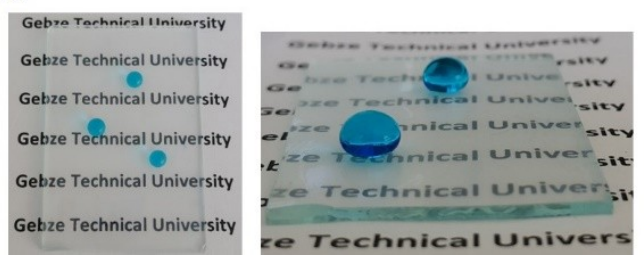

Figure 3. I. SEM pictures and water droplets of PDMS-silica composite films prepared with 63k PDMS and three different hydrophobic and hydrophilic fumed silica particles: (a) FS (b) H18, (c) H15 and (c) N20 (Silica concentration is $4 \% \mathrm{w} / \mathrm{v}$ ). II. Photographs of superhydrophobic PDMS-silica composite film (63k PDMS, 4\% w/v FS) coated glass slide (water droplets were dyed with methylene blue).

\section{CONCLUSIONS}

Summarizing, in this paper, we have demonstrated the preparation of covalently attached superhydrophobic PDMS/silica composite coatings on activated glass slides via an easy and straightforward spin-coating process. SEM and contact angle measurements were used to characterize the morphology and the wettability of the surfaces. The superhydrophobic coatings were obtained when PDMS modified fumed silica (FS and $\mathrm{H} 18$ ) were used, and the concentration of the fumed silica was $4 \% \mathrm{w} / \mathrm{v}$ whereas the superhydrophobic surfaces were not obtained when hydrophilic (N2O) and dimethylsiloxy-modified (H15) fumed silica were used due to the high CAH. To obtain a superhydrophobic surface using PDMS and fumed silica via a spin coating process, the type and the concentration of the fumed silica need to be optimized.

\section{ACKNOWLEDGMENTS}

This research is supported by Gebze Technical University Research Fund. (Project Number: GTU 2016-A17) and originates from Alper Nerat's MS Thesis (\#523031). We thank Mr. Ahmet Nazım in the Materials Science and Engineering Department of Gebze Technical University for taking the SEM images.

\section{REFERENCES}

1. Barthlott W, Neinhuis C. Purity of the sacred lotus, or escape from contamination in biological surfaces. Planta 1997; 202 (1): 1-8.

2. Chen W, Fadeev AY, Hsieh MC, Oner D, Yougblood J et al. Ultrahydrophobic and ultralyophobic surfaces: some comments and examples. Langmuir 1999; 15 (10): 33959.

3. Erbil HY, Demirel AL, Avci Y, Mert O. Transformation of a simple plastic into a superhydrophobic surface. Science 2003; 299 (5611): 1377-80.

4. Yan YY, Gao N, Barthlott W. Mimicking natural superhydrophobic surfaces and grasping the wetting process: a review on recent progress in preparing superhydrophobic surfaces, Advances in Colloid and Interface Science 2011; 169 (2): 80-105.

5. Roach $P$, Shirtcliffe NJ, Newton MI. Progress in superhyrophobic surface development. Soft Matter 2008; 4 (2); 224-40.

6. Feng L, Li SH, Li YS, Li HJ, Zhang LJ et al. Superhydrophobic surfaces: from natural to artificial. Advance Materials 2002; 14 (24): 1857-60.

7. Liu K, Tian Y, Jiang L. Bio-inspired superoleophobic and smart materials: design, fabrication, and application. Progress in Materials Science 2013; 58 (4): 503-64.

8. Cassie ABD, Baxter S. Wettability of porous surfaces. Transactions of the Faraday Society 1944; 40: 546-51.

9. Erbil HY. The debate on the dependence of apparent contact angles on drop contact area or three-phase contact line: A review. Surface Science Reports 2014; 69 (4): 32565

10. Atici EG, Kasapgil E, Anac I, Erbil HY. Methyltrichlorosilane polysiloxane filament growth on glass using low cost solvents and comparison with gas phase reactions. Thin Solid Films 2016; 616: 101-10.

11. Kasapgil E, Atici EG, Cicek R, Anac I, Erbil HY. Superhydrophobic polysiloxane filament growth on nonactivated polymer coatings. RSC Advances 2016; 6: 74921-8.

12. Tuteja A, Choi W, Ma M, Mabry JM, Mazzela SA, Rutlege GC, McKinley $\mathrm{GH}$, Cohen RH. Designing 
superoleophobic surfaces. Science 2007; 318 (5856): 1618-22.

13. Jung YC, Bhushan B. Mechanically durable carbon nanotube-composite hierarchical structures with superhydrophobicity, self-cleaning, and low-drag. ACS Nano 2009; 3: 4155-63.

14. Manoudis PN, Karapanagiotis I, Tsakalof A, Zuburtikubis I, Panayiotou C. Superhydrophobic composite films produced on various substrates. Langmuir 2008; 24 (19): 11225-32.

15. Hsieh CT, Chen JM, Kuo RR, Lin TS, Wu CF. Influence of surface roughness on water- and oil-repellent surfaces coated with nanoparticles. Applied Surface Science. 2005; 240(1):318-26.

16. Chibowski $\mathrm{E}$, Holysz $\mathrm{L}$, Terpolowski $\mathrm{K}$, Jurak $\mathrm{M}$. Investigation of superhydrophobic effect of PMMA layers with different fillers deposited on glass support. Colloids and Surfaces A: Physicochem. Eng. Aspects. 2006; 291 (13): 181-90.

17. Yang J, Pi $P$, Wen $X$, Zheng $D, X u$ et al. A novel method to fabricate superhydrophobic surfaces based on well-defined mulberry-like particles and self-assembly of polydimethylsiloxane. Applied Surface Science 2009; 255 (6): 3507-12.

18. Yuce $Y M$, Demirel AL, Menzel F. Tuning the surface hydrophobicity of polymer/ nanoparticle composite films in the Wenzel regime by composition. Langmuir, 2005; 21(11): 5073-8.

19. Ke Q, Fu W, Jin $\mathrm{H}$, Zhang $\mathrm{L}$, Tang $\mathrm{T}$ et al. Fabrication of mechanically robust superhydrophobic surfaces based on silica micro-nanoparticles and polydimethylsiloxane. Surface \& Coatings Technology 2011; 205 (21-22): 49104.

20. Chang $\mathrm{H}$, Tu $\mathrm{K}$, Wang $\mathrm{X}$, Liu J. Fabrication of mechanically durable superhydrophobic wood surfaces using polydimethylsiloxane and silica nanoparticles. RSC Advances 2015: 5 (39): 30647-53.

21. Soz CK, Yilgor E, Yilgor I. Influence of the average surface roughness on the formation of superhydrophobic polymer surfaces through spin-coating with hydrophobic fumed silica. Polymer 2015; 62: 118-28.

22. Soz CK, Yilgor E, Yilgor I. Simple processes for the preparation of superhydrophobic polymer surfaces. Polymer 2016; 99: 580-93. doi: $10.1016 /$ j.polymer.2016.07.051

23. Yilgor I, Bilgin S, Isik M, Yilgor E. Facile preparation of superhydrophobic polymer surfaces. Polymer 2012; 53 (6): $1180-8$

24. Cai R, Glinel K, De Smet D, Vanneste M, Mannu N et al. Environmentally friendly super-water-repellent fabrics prepared from water-based suspensions. ACS Applied Materials and Interfaces 2018; 10 (18): 15346-51.

25. Fourmentin A, Galy J, Charlot A, Gerard J-F. Bioinspired silica-containing polyurethane-acrylate films: Towards superhydrophobicity with tunable water adhesion. Polymer 2018; 155: 1-12.

26. Sriram A, Kumar A. Separation of oil-water via porous PMMA/SiO2 nanoparticles superhydrophobic surface. Colloids and Surfaces A: Physicochemical and Engineering Aspects 2019; 563: 271-9.

27. Yang J, Pi P, Wen X, Zheng $D, X u$ et al. A novel method to fabricate superhydrophobic surfaces based on well-defined mulberry-like particles and self-assembly of polydimethylsiloxane. Applied Surface Science 2009; 255 (6): $3507-12$.

28. Krumpfer JW, McCarthy TJ. Rediscovering silicones: "Unreactive" silicones react with inorganic surfaces. Langmuir 2011; 27 (18): 11514-9 